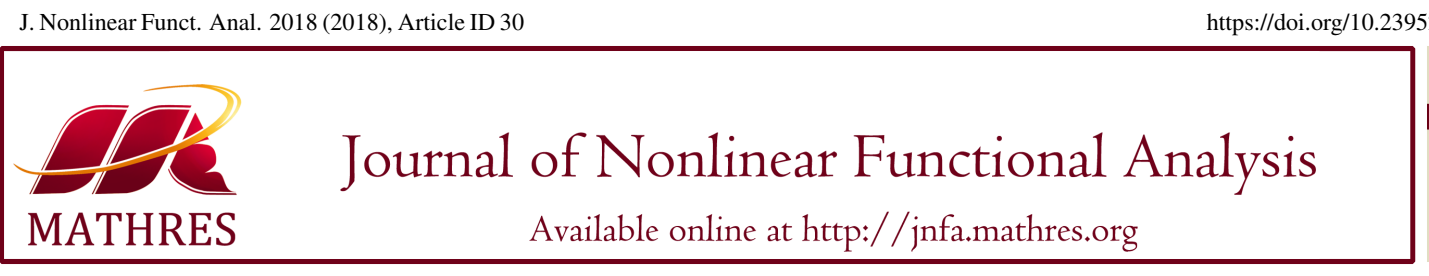

\title{
ENERGY ESTIMATE FOR IMPULSIVE FRACTIONAL ADVECTION DISPERSION EQUATIONS IN ANOMALOUS DIFFUSIONS
}

\author{
NADER BIRANVAND, AMJAD SALARI*
}

Faculty of Sciences, Imam Ali University, Tehran, Iran

\begin{abstract}
This paper deals with the existence and energy estimates of solutions for a class of impulsive fractional advection dispersion equations in anomalous diffusions, while the nonlinear part of the problem admits some hypotheses on the behavior at origin or perturbation property. In particular, for a precise localization of the parameter, the existence of a non-zero solution is established requiring the sublinearity of nonlinear part at origin and infinity. We also consider the existence of solutions for our problem under algebraic conditions with the classical Ambrosetti-Rabinowitz. By combining two algebraic conditions on the nonlinear term which guarantees the existence of two solutions as well as applying the mountain pass theorem given by Pucci and Serrin, we establish the existence of the third solution for our problem. Moreover, concrete examples of applications are also provided.
\end{abstract}

Keywords. Clasical solution; Critical point theory; Fractional differential equation; Variational methods; Weak solution.

2010 Mathematics Subject Classification. 34B37, 58E05, 58E30.

\section{INTRODUCTION}

In [1], Risken introduced an advection-dispersion equation to describe the Brownian motion of particles

$$
\frac{\partial C(x, t)}{\partial t}=\left[-v \frac{\partial}{\partial x}+D \frac{\partial^{2}}{\partial x^{2}}\right] C(x, t)
$$

where $C(x, t)$ is a concentration field of space variable $x$ at time $t, D>0$ is the diffusion coefficient and $v>0$ is the drift coefficient. Many laboratory data [2,3] and numerical experiments [4] indicate that solutes moving through a highly heterogeneous aquifer violate the basic assumptions of the local second order theories because of the large deviations due to the stochastic process of Brownian motion. According to [2], an anomalous dispersion process should be described by the following advectiondispersion equation containing the left and the right fractional differential operators

$$
\frac{\partial C(x, t)}{\partial t}=-v \frac{\partial C(x, t)}{\partial x}+D j \frac{\partial^{\gamma} C(x, t)}{\partial x^{\gamma}}+D(1-j) \frac{\partial^{\gamma} C(x, t)}{\partial(-x)^{\gamma}},
$$

\footnotetext{
${ }^{*}$ Corresponding author.
}

E-mail addresses: nabiranvand@gmail.com (N. Biranvand), amjads45@yahoo.com (A. Salari).

Received April 24, 2018; Accepted August 27, 2018. 
where $C$ is the expected concentration field of space variable $x$ at time $t, v$ is a constant mean velocity, $x$ is the distance in the direction of the mean velocity, $D$ is a constant dispersion coefficient, $0 \leq j \leq 1$ describes the skewness of the transport process, and $\gamma$ is the order of left and right fractional differential operators (see [2, Appendix] for details about left and right fractional differential operators). Especially, if $\gamma=2$, the dispersion operator reduces to the classical advection-dispersion operator and (1.1) becomes the classical advection-dispersion equation. On the other hand, if $j=\frac{1}{2},(1.1)$ describes symmetric transitions. Define an equivalent Riesz potential symmetric operator [5]

$$
2 \nabla^{\gamma} \equiv D_{+}^{\gamma}+D_{-}^{\gamma}
$$

which gives the mass balance equation for the symmetric fractional advection dispersion

$$
\frac{\partial C(x, t)}{\partial t}=-v \nabla C(x, t)+D \nabla^{\gamma} C(x, t) .
$$

Fractional differential equations (FDEs) is a simplification of ordinary differential equations and integration into arbitrary non-integer orders. FDEs have recently established themselves as precious tools in modeling many events in different fields of science and engineering. We can also observe plentiful applications in such fields as electrochemistry, chemistry, electromagnetic, mechanics, biology, electricity, economics, polymer rheology, control theory, regular variation in thermodynamics, signal and image processing, wave propagation, aerodynamics, electrodynamics of complex medium, blood flow phenomena, biophysics, viscoelasticity and damping, etc. (see $[5,6,7,8,9]$ ). There has also been important advances in theory of fractional calculus and fractional ordinary and partial differential equations recently; see $[10,11]$ as an example. Many researchers have explored the existence of solutions for nonlinear FDEs with various tools such as fixed-point theorems, the method of upper and lower solutions, critical point theory, the topological degree theory, and variational methods; see, for instance, $[12,13,14,15,16]$ and the references therein. This type of equations can be used to simulate anomalous diffusion in fractal media. They established the existence and uniqueness of local and global mild solutions and proved the existence and regularity of classical solutions.

On the other hand, impulsive differential equations have become important in recent years as mathematical models of phenomena in both the physical and social sciences. For example, many biological phenomena involving thresholds, bursting rhythm models in medicine and biology, optimal control models in economics and frequency modulated systems, do exhibit impulsive effects. For the background and applications of the theory of impulsive differential equations to different areas, we refer the reader to the classical monograph [17]. For the general aspects of impulsive differential equations, we refer the reader to $[18,19,20]$. The existence of multiple solutions of impulsive problems has been studied also using the variational methods and critical point theory (see [21]). Both FDEs and impulsive differential equations have drawn intense attention from researchers in the last decades due to the numerous applications. The idea that combining these two classes of differential equations may yield an interesting and promising object of investigation, viz., impulsive FDEs, prompted numerous papers. For the recent developments in theory and applications of impulsive FDEs, we refer the reader to the papers $[22,23]$ and the references therein. Impulsive problems for fractional equations have been treated by topological methods in $[24,25,26,27]$. In [21, 28], based on variational methods and critical point theory the authors studied the existence and multiplicity of solutions for the problem $\left(D_{\lambda}\right)$, in the case $h(x)=0$ for all $x \in \mathbb{R}$. 
We also cite the papers [29, 30, 31, 32, 33] in which fractional systems have been studied. In [32, 33], through variational methods and critical point theory the existence of multiple solutions for coupled systems of nonlinear fractional differential equations was analyzed. In [31], using Ricceri's Variational Principle, the existence of one weak solution for a class of fractional differential systems was argued. In [30], employing Ricceri's Variational Principle, the existence of an infinite number of weak solutions for a class of impulsive fractional differential systems was guaranteed. In [29], using variational methods and critical point theory, the multiplicity results of solutions for a class of impulsive fractional differential systems was established.

In this paper, due to the researches above, we are interested in the existence results and energy estimates of solutions for for the following impulsive nonlinear fractional boundary value problem

$$
\begin{gathered}
{ }_{t} D_{T}^{\alpha}\left({ }_{0}^{c} D_{t}^{\alpha} u(t)\right)+a(t) u(t)=\lambda f(t, u(t)), \quad t \neq t_{j}, \text { a.e. } t \in[0, T], \\
\Delta\left({ }_{t} D_{T}^{\alpha-1}\left({ }_{0}^{c} D_{t}^{\alpha} u\right)\right)\left(t_{j}\right)=\lambda I_{j}\left(u\left(t_{j}\right)\right), \quad j=1, \ldots n, \\
u(0)=u(T)=0,
\end{gathered}
$$

where $\alpha \in(1 / 2,1], a \in \mathrm{C}([0, T])$ such that there are $a_{1}, a_{2}>0$ such that $0<a_{1} \leq a(t) \leq a_{2}, \lambda>0, f$ : $[0, T] \times \mathbb{R} \rightarrow \mathbb{R}$ is an $\mathrm{L}^{1}$-Carathéodory function, $0=t_{0}<t_{1}<\ldots<t_{n}<t_{n+1}=T, \Delta\left({ }_{t} D_{T}^{\alpha-1}\left({ }_{0}^{c} D_{t}^{\alpha} u\right)\right)\left(t_{j}\right)=$ ${ }_{t} D_{T}^{\alpha-1}\left({ }_{0}^{c} D_{t}^{\alpha} u\right)\left(t_{j}^{+}\right)-{ }_{t} D_{T}^{\alpha-1}\left({ }_{0}^{c} D_{t}^{\alpha} u\right)\left(t_{j}^{-}\right)$and $I_{j}: \mathbb{R} \rightarrow \mathbb{R}, j=1, \ldots, n$ are Lipschitz continuous functions with the Lipschitz constants $L_{j}>0$, i.e.,

$$
\left|I_{j}\left(x_{1}\right)-I_{j}\left(x_{2}\right)\right| \leq L_{j}\left|x_{1}-x_{2}\right|
$$

for every $x_{1}, x_{2} \in \mathbb{R}$ and $I_{j}(0)=0$.

The main result of this paper ensures the existence of exact values of the parameter $\lambda$ for which problem $\left(D_{\lambda}\right)$ admits at least one/two/three non-zero weak solutions. Several special cases of the main results and examples are also given. We also refer the reader to $[34,35]$ for some related results in this subject.

\section{PRELIMINARIES}

In this section, we will introduce several basic definitions, notations, lemmas, and propositions used all over this paper.

Definition 2.1 ([7]). For a function $f$ defined on $[a, b]$ and $\alpha>0$, the left and right Riemann-Liouville fractional integrals of order $\alpha$ for the function $f$ are defined by

$$
\begin{array}{ll}
{ }_{a} D_{t}^{-\alpha} f(t)=\frac{1}{\Gamma(\alpha)} \int_{a}^{t}(t-s)^{\alpha-1} f(s) \mathrm{d} s, & t \in[a, b], \\
{ }_{t} D_{b}^{-\alpha} f(t)=\frac{1}{\Gamma(\alpha)} \int_{t}^{b}(s-t)^{\alpha-1} f(s) \mathrm{d} s, & t \in[a, b],
\end{array}
$$

while the right-hand sides are point-wise defined on $[a, b]$, where $\Gamma(\alpha)$ is the gamma function.

Definition 2.2 ([7]). Let $a, b \in \mathbb{R}$ and $\mathrm{AC}([a, b])$ be the space of absolutely continuous functions on $[a, b]$. For $0<\alpha \leq 1, f \in \mathrm{AC}([a, b])$ left and right Riemann-Liouville and Caputo fractional derivatives are defined by:

$$
{ }_{a} D_{t}^{\alpha} f(t) \equiv \frac{\mathrm{d}}{\mathrm{d} t}{ }_{a} D_{t}^{\alpha-1} f(t)=\frac{1}{\Gamma(1-\alpha)} \frac{\mathrm{d}}{\mathrm{d} t} \int_{a}^{t}(t-s)^{-\alpha} f(s) \mathrm{d} s
$$




$$
\begin{gathered}
{ }_{t} D_{b}^{\alpha} f(t) \equiv-\frac{\mathrm{d}}{\mathrm{d} t}{ }_{t} D_{b}^{\alpha-1} f(t)=-\frac{1}{\Gamma(1-\alpha)} \int_{t}^{b}(s-t)^{-\alpha} f(s) \mathrm{d} s, \\
{ }_{a}^{c} D_{t}^{\alpha} f(t) \equiv{ }^{c} D_{a^{+}}^{\alpha} f(t):={ }_{a} D_{t}^{\alpha-1} f^{\prime}(t)=\frac{1}{\Gamma(1-\alpha)} \int_{a}^{t}(t-s)^{-\alpha} f^{\prime}(s) \mathrm{d} s
\end{gathered}
$$

and

$$
{ }_{t}^{c} D_{b}^{\alpha} f(t) \equiv{ }^{c} D_{b-}^{\alpha} f(t):=-{ }_{t} D_{b}^{\alpha-1} f^{\prime}(t)=-\frac{1}{\Gamma(1-\alpha)} \int_{t}^{b}(s-t)^{-\alpha} f^{\prime}(s) \mathrm{d} s
$$

where $\Gamma(\alpha)$ is the gamma function. Note that when $\alpha=1,{ }_{a}^{c} D_{t}^{1} f(t)=f^{\prime}(t)$ and ${ }_{t}^{c} D_{b}^{1} f(t)=-f^{\prime}(t)$

We have the following property of fractional integration.

Proposition $2.3([5,7])$.

$$
\int_{a}^{b}\left[{ }_{a} D_{t}^{-\gamma} f(t)\right] g(t) \mathrm{d} t=\int_{a}^{b}\left[{ }_{t} D_{b}^{-\gamma} g(t)\right] f(t) \mathrm{d} t, \quad \gamma>0,
$$

provided that $f \in \mathrm{L}^{p}\left([a, b], \mathbb{R}^{N}\right), g \in \mathrm{L}^{q}\left([a, b], \mathbb{R}^{N}\right)$ and $p \geq 1, q \geq 1,1 / p+1 / q \leq 1+\gamma$ or $p \neq 1, q \neq 1$, $1 / p+1 / q=1+\gamma$.

To create suitable function spaces and apply critical point theory to explore the existence of solutions for problem $\left(D_{\lambda}\right)$, we require the following essential notations and findings which will be used in establishing our main results.

Let $0<\alpha \leq 1$ and $1<p<\infty$. Let $\mathrm{E}_{0}^{\alpha, p}(0, T)$ be a Banach space, which is closure of $\mathrm{C}_{0}^{\infty}([0, T])$ with respect to the norm

$$
\|u\|_{\mathrm{E}_{0}^{\alpha, p}(0, T)}^{p}=\left\|{ }_{a}^{c} D_{t}^{\alpha} u(t)\right\|_{\mathrm{L}^{p}(0, T)}^{p}+\|u\|_{\mathrm{L}^{p}(0, T)}^{p} .
$$

It is an established fact that $\mathrm{E}_{0}^{\alpha, p}(0, T)$ is a reflexive and separable Banach space (see [36, Proposition 3.1]). In short $\mathrm{E}_{0, T}^{\alpha, 2}=\mathrm{E}^{\alpha}$, and by $\|\cdot\|$ and $\|\cdot\|_{\infty}$ the norms in $\mathrm{L}^{2}(0, T)$ and $\mathrm{C}([0, T])$ :

$$
\begin{gathered}
\|u\|^{2}=\int_{0}^{T}|u(t)|^{2} \mathrm{~d} t, \quad u \in \mathrm{L}^{2}(0, T), \\
\|u\|_{\infty} \max _{t \in[0, T]}|u(t)|, \quad u \in \mathrm{C}([0, T]) .
\end{gathered}
$$

$\mathrm{E}^{\alpha}$ is a Hilbert space with inner product

$$
(u, v)_{\alpha}=\int_{0}^{T}\left({ }_{0}^{c} D_{t}^{\alpha} u(t){ }_{0}^{c} D_{t}^{\alpha} v(t)+u(t) v(t)\right) \mathrm{d} t
$$

and the norm

$$
\|u\|_{\alpha}^{2}=\int_{0}^{T}\left(\left.{ }_{0}^{c} D_{t}^{\alpha} u(t)\right|^{2}+|u(t)|^{2}\right) \mathrm{d} t
$$

If $a \in \mathrm{C}([0, T])$ and there are two positive constants $a_{1}$ and $a_{2}$, so that $0<a_{1} \leq a(t) \leq a_{2}$, an equivalent norm in $E^{\alpha}$ is

$$
\|u\|_{a, \alpha}^{2}=\int_{0}^{T}\left(\left|{ }_{0}^{c} D_{t}^{\alpha} u(t)\right|^{2} d t+a(t)|u(t)|^{2}\right) \mathrm{d} t .
$$

Proposition 2.4 ([36]). Let $0<\alpha \leq 1$. For $u \in \mathrm{E}^{\alpha}$, we have

$$
\|u\| \leq \frac{T^{\alpha}}{\Gamma(\alpha+1)}\left\|_{0}^{c} D_{t}^{\alpha} u\right\|
$$

In addition, for $\frac{1}{2}<\alpha \leq 1$,

$$
\|u\|_{\infty} \leq \frac{T^{\alpha-1 / 2}}{\Gamma(\alpha)(2 \alpha-1)^{1 / 2}}\left\|_{0}^{c} D_{t}^{\alpha} u\right\| .
$$


By (2.1), we can take $\mathrm{E}^{\alpha}$ with the norm

$$
\|u\|_{0, \alpha}=\left(\int_{0}^{T}\left|{ }_{0}^{c} D_{t}^{\alpha} u(t)\right|^{2} \mathrm{~d} t\right)^{1 / 2}=\left\|_{0}^{c} D_{t}^{\alpha} u\right\|, \quad \forall u \in \mathrm{E}^{\alpha} .
$$

By Proposition 2.4, when $\alpha>1 / 2$, for every $u \in \mathrm{E}^{\alpha}$, we have

$$
\|u\|_{\infty} \leq k\left(\left.\int_{0}^{T}{ }_{0}^{c} D_{t}^{\alpha} u(t)\right|^{2} \mathrm{~d} t\right)^{1 / 2}=k\|u\|_{0, \alpha}<k\|u\|_{a, \alpha},
$$

where

$$
k=\frac{T^{\alpha-\frac{1}{2}}}{\Gamma(\alpha) \sqrt{2 \alpha-1}}
$$

Now, by setting $L:=\sum_{i=1}^{n} L_{j}$, we put

$$
\begin{aligned}
& C_{1}:=\frac{1}{2}\left(1-L T k^{2}\right), \\
& C_{2}:=\frac{1}{2}\left(1+L T k^{2}\right) .
\end{aligned}
$$

We suppose that the Lipschitz constant $L>0$ of the function $h$ satisfies the condition $L T k^{2}<1$.

Here we give the definition of weak and classical solutions for problem $\left(D_{\lambda}\right)$ as below.

Definition 2.5. A function $u \in \mathrm{E}^{\alpha}$ is said to be a weak solution of $\left(D_{\lambda}\right)$, if for every $v \in \mathrm{E}^{\alpha}$,

$$
\begin{aligned}
\int_{0}^{T} & {\left.\left[{ }_{0}^{c} D_{t}^{\alpha} u(t)\right)\left({ }_{0}^{c} D_{t}^{\alpha} v(t)\right)+a(t) u(t) v(t)\right] \mathrm{d} t+\lambda \sum_{j=1}^{n} I_{j}\left(u\left(t_{j}\right)\right) v\left(t_{j}\right) } \\
& =\lambda \int_{0}^{T} f(t, u(t)) v(t) \mathrm{d} t .
\end{aligned}
$$

Definition 2.6. A function

$$
u \in\left\{u \in \mathrm{AC}([0, T]): \int_{t_{j}}^{t_{j+1}}\left(\left|{ }_{0}^{c} D_{t}^{\alpha} u(t)\right|^{2}+|u(t)|^{2}\right) \mathrm{d} t<\infty, j=0, \ldots n\right\}
$$

is said to be a classical solution of problem $\left(D_{\lambda}\right)$ if

$$
{ }_{t} D_{T}^{\alpha}\left({ }_{0}^{c} D_{t}^{\alpha} u(t)\right)+a(t) u(t)=\lambda f(t, u(t))+h(u(t)), \quad \text { a.e. } t \in[0, T] \backslash\left\{t_{1}, \ldots, t_{n}\right\},
$$

the limits ${ }_{t} D_{T}^{\alpha-1}\left({ }_{0}^{c} D_{t}^{\alpha} u\right)\left(t_{j}^{+}\right)$and ${ }_{t} D_{T}^{\alpha-1}\left({ }_{0}^{c} D_{t}^{\alpha} u\right)\left(t_{j}^{-}\right)$exist, $\Delta\left({ }_{t} D_{T}^{\alpha-1}\left({ }_{0}^{c} D_{t}^{\alpha} u\right)\right)\left(t_{j}\right)=\mu I_{j}\left(u\left(t_{j}\right)\right)$ and $u(0)=$ $u(T)=0$.

Lemma 2.7 ([21, Lemma 2.1]). A function $u \in \mathrm{E}^{\alpha}$ is a weak solution of $\left(D_{\lambda}\right)$ if and only if it is a classical solution of $\left(D_{\lambda}\right)$.

We refer the reader to $[37,38]$ for the following notations and results. Let $X$ be a real Banach space. We say that a continuously Gâteaux differentiable functional $J: X \rightarrow \mathbb{R}$ satisfies the Palais-Smale condition (in short (PS)-condition) if any sequence $\left\{u_{n}\right\}$ such that

$\left(\mathrm{j}_{1}\right)\left\{J\left(u_{n}\right)\right\}$ is bounded,

$\left(\mathrm{j}_{2}\right) \lim _{n \rightarrow \infty}\left\|J^{\prime}\left(u_{n}\right)\right\|_{X^{*}}=0$,

has a convergent subsequence.

Let $\Phi, \Psi: X \rightarrow \mathbb{R}$ be two continuously Gâteaux differentiable functions. Set

$$
J=\Phi-\Psi
$$

and fix $r_{1}, r_{2} \in[-\infty,+\infty]$ with $r_{1}<r_{2}$. We say that $J$ satisfies the Palais-Smale condition cut off lower at $r_{1}$ and upper at $r_{2}$ (in short ${ }^{\left[r_{1}\right]}(\mathrm{PS})^{\left[r_{2}\right]}$-condition) if any sequence $\left\{u_{n}\right\}$ satisfying $\left(\mathrm{j}_{1}\right),\left(\mathrm{j}_{2}\right)$ and 
$\left(\mathrm{j}_{3}\right) r_{1}<\Phi\left(u_{n}\right)<r_{2}, \forall n \in \mathbb{N}$,

has a convergent subsequence.

Clearly, if $r_{1}=-\infty$ and $r_{2}=+\infty$, it coincides with the classical (PS)-condition. Moreover, if $r_{1}=-\infty$ and $r_{2} \in \mathbb{R}$, it is denoted by (PS) ${ }^{\left[r_{2}\right]}$, while if $r_{1} \in \mathbb{R}$ and $r_{2}=+\infty$, it is denoted by ${ }^{\left[r_{1}\right]}$ (PS). Furthermore, if $J$ satisfies ${ }^{\left[r_{1}\right]}(\mathrm{PS})^{\left[r_{2}\right]}$-condition, it satisfies ${ }^{\left[\rho_{1}\right]}(\mathrm{PS})^{\left[\rho_{2}\right]}$-condition for all $\rho_{1}, \rho_{2} \in[-\infty,+\infty]$ such that $r_{1} \leq \rho_{1}<\rho_{2} \leq r_{2}$.

In particular, we deduce that if $J$ satisfies the classical (PS)-condition, then it satisfies ${ }^{\left[\rho_{1}\right]}(\mathrm{PS})^{\left[\rho_{2}\right]_{-}}$ condition for all $\rho_{1}, \rho_{2} \in[-\infty,+\infty]$ with $\rho_{1}<\rho_{2}$. For $r \in \mathbb{R}$ and $r_{1}, r_{2} \in \mathbb{R}$ with $r_{1}<r_{2}$, we set

$$
\begin{aligned}
& \rho(r)=\sup _{v \in \Phi^{-1}(r,+\infty)} \frac{\Psi(v)-\sup _{u \in \Phi^{-1}(-\infty, r]} \Psi(u)}{\Phi(v)-r}, \\
& \beta\left(r_{1}, r_{2}\right):=\inf _{v \in \Phi^{-1}\left(r_{1}, r_{2}\right)} \frac{\sup _{u \in \Phi^{-1}\left(r_{1}, r_{2}\right)} \Psi(u)-\Psi(v)}{r_{2}-\Phi(v)},
\end{aligned}
$$

and

$$
\rho_{2}\left(r_{1}, r_{2}\right):=\sup _{v \in \Phi^{-1}\left(r_{1}, r_{2}\right)} \frac{\Psi(v)-\sup _{u \in \Phi^{-1}\left(-\infty, r_{1}\right]} \Psi(u)}{\Phi(v)-r_{1}} .
$$

In the proof of our main results, we will apply the following two Theorems.

Theorem 2.8. [39, Theorem 5.1] (see also [37, 38]) Let $X$ be a real Banach space and let $\Phi, \Psi$ : $X \rightarrow \mathbb{R}$ be two continuously Gâteaux differentiable functions. Assume that there exist $r_{1}, r_{2} \in \mathbb{R}$ with $r_{1}<r_{2}$, such that $\beta\left(r_{1}, r_{2}\right)<\rho_{2}\left(r_{1}, r_{2}\right)$, where $\beta$ and $\rho_{2}$ are given by (2.5) and (2.6), and for each $\lambda \in$ $\left(\frac{1}{\rho_{2}\left(r_{1}, r_{2}\right)}, \frac{1}{\beta\left(r_{1}, r_{2}\right)}\right)$, the function $J_{\lambda}:=\Phi-\lambda \Psi$ satisfies ${ }^{\left[r_{1}\right]}(\mathrm{PS})^{\left[r_{2}\right]}$ condition. Then, $\forall \lambda \in\left(\frac{1}{\rho_{2}\left(r_{1}, r_{2}\right)}, \frac{1}{\beta\left(r_{1}, r_{2}\right)}\right)$ there exists $u_{0, \lambda} \in \Phi^{-1}\left(r_{1}, r_{2}\right)$ such that $J_{\lambda}\left(u_{0, \lambda}\right) \leq J_{\lambda}(u)$ for all $u \in \Phi^{-1}\left(r_{1}, r_{2}\right)$ and $J_{\lambda}^{\prime}\left(u_{0, \lambda}\right)=0$.

Theorem 2.9. [39, Corolary 5.1] Let $X$ be a real Banach space and let $\Phi, \Psi: X \rightarrow \mathbb{R}$ be two continuously Gâteaux differentiable function. Put

$$
\beta^{*}:=\liminf _{r \rightarrow+\infty} \frac{\sup _{u \in \Phi^{-1}(-\infty, r)} \Psi(u)}{r}
$$

and assume that there is $\bar{r} \in \mathbb{R}$ such that $\rho(\bar{r})>\beta^{*}$ where $\rho$ is given by (2.4). Moreover, assume that for each $\lambda \in\left(\frac{1}{\rho(\bar{r})}, \frac{1}{\beta^{*}}\right)$ the function $J_{\lambda}:=\Phi-\lambda \Psi$ satisfies ${ }^{[\bar{r}]}(\mathrm{PS})^{[r]}$-condition for all $r>\bar{r}$. Then there is $r_{2}>\bar{r}$ such that for each $\lambda \in\left(\frac{1}{\rho(\bar{r})}, \frac{1}{\beta^{*}}\right)$, there is $u_{0, \lambda} \in \Phi^{-1}\left(\bar{r}, r_{2}\right)$ such that $J_{\lambda}\left(u_{0, \lambda}\right) \leq J_{\lambda}(u)$ for all $u \in \Phi^{-1}\left(\bar{r}, r_{2}\right)$ and $J_{\lambda}^{\prime}\left(u_{0, \lambda}\right)=0$.

Corresponding to the functions $f, h$ and $I_{j}, j=1 \ldots, n$, we introduce the functions $F:[0, T] \times \mathbb{R} \longrightarrow \mathbb{R}$ and $J_{j}:[0, T] \times \mathbb{R} \longrightarrow \mathbb{R}, j=1, \ldots, n$, respectively, as follows

$$
F(t, \xi):=\int_{0}^{\xi} f(t, x) \mathrm{d} x, \text { for all } \xi \in \mathbb{R}
$$

and

$$
J_{j}(x)=\int_{0}^{x} I_{j}(\xi) \mathrm{d} \xi, \quad j=1, \ldots, n \quad \text { for every } x \in \mathbb{R} .
$$

Throughout this paper, we consider the following conditions on impulsive terms

$(\mathscr{H})$ let $I_{j} \geq 0$ for all $j=1, \ldots, n$.

We need the following proposition for existence our main results. 
Proposition 2.10. Let $S: \mathrm{E}^{\alpha} \longrightarrow\left(\mathrm{E}^{\alpha}\right)^{*}$ be the operator defined by

$$
\left.S(u)(v)=\int_{0}^{T}\left[{ }_{0}^{c} D_{t}^{\alpha} u(t)\right)\left({ }_{0}^{c} D_{t}^{\alpha} v(t)\right)+a(t) u(t) v(t)\right] \mathrm{d} t
$$

for every $u, v \in \mathrm{E}^{\alpha}$. Then, $S$ admits a continuous inverse on $\left(\mathrm{E}^{\alpha}\right)^{*}$.

Proof. It is obvious that

$$
S(u)(u)=\int_{0}^{T}\left(\left|{ }_{0}^{c} D_{t}^{\alpha} u(t)\right|^{2}+a(t)|u(t)|^{2}\right) \mathrm{d} t \geq\|u\|_{a, \alpha}^{2} .
$$

It follows that $S$ is coercive. Hence,

$$
\begin{aligned}
\langle S(u)-S(v), u-v\rangle & =\int_{0}^{T}\left(\left|{ }_{0}^{c} D_{t}^{\alpha}(u(t)-v(t))\right|^{2}+a(t)|u(t)-v(t)|^{2}\right) \mathrm{d} t \\
& \geq\|u-v\|_{a, \alpha}^{2}>0
\end{aligned}
$$

for every $u, v \in \mathrm{E}^{\alpha}$, which means that $S$ is strictly monotone. Moreover, since $\mathrm{E}^{\alpha}$ is reflexive, for $u_{n} \longrightarrow u$ strongly in $\mathrm{E}^{\alpha}$ as $n \rightarrow+\infty$, one has $S\left(u_{n}\right) \rightarrow S(u)$ weakly in $\left(E^{\alpha}\right)^{*}$ as $n \rightarrow \infty$. Hence, $S$ is demicontinuous. By [40, Theorem 26.A(d)], the inverse operator $S^{-1}$ of $S$ exists and it is continuous. Indeed, let $e_{n}$ be a sequence of $\left(\mathrm{E}^{\alpha}\right)^{*}$ such that $e_{n} \rightarrow e$ strongly in $\left(\mathrm{E}^{\alpha}\right)^{*}$ as $n \rightarrow \infty$. Let $u_{n}$ and $u$ in $\mathrm{E}^{\alpha}$ such that $S^{-1}\left(e_{n}\right)=u_{n}$ and $S^{-1}(e)=u$. Taking into account that $S$ is coercive, one has that the sequence $u_{n}$ is bounded in the reflexive space $\mathrm{E}^{\alpha}$. For a suitable subsequence, we have $u_{n} \rightarrow \hat{u}$ weakly in $\mathrm{E}^{\alpha}$ as $n \rightarrow \infty$, which concludes

$$
\left\langle S\left(u_{n}\right)-S(u), u_{n}-\hat{u}\right\rangle=\left\langle e_{n}-e, u_{n}-\hat{u}\right\rangle=0 .
$$

If $u_{n} \rightarrow \hat{u}$ weakly in $\mathrm{E}^{\alpha}$ as $n \rightarrow+\infty$ and $S\left(u_{n}\right) \rightarrow S(\hat{u})$ strongly in $\left(\mathrm{E}^{\alpha}\right)^{*}$ as $n \rightarrow+\infty$, one has $u_{n} \rightarrow \hat{u}$ strongly in $\mathrm{E}^{\alpha}$ as $n \rightarrow+\infty$. Since $S$ is continuous, one has $u_{n} \rightarrow \hat{u}$ weakly in $\mathrm{E}^{\alpha}$ as $n \rightarrow+\infty$ and $S\left(u_{n}\right) \rightarrow S(\hat{u})=S(u)$ strongly in $\left(\mathrm{E}^{\alpha}\right)^{*}$ as $n \rightarrow+\infty$. Hence, taking into account that $S$ is an injection, we have $u=\hat{u}$.

\section{MAin Results}

In this section, we formulate our main results. Put

$$
A(\alpha):=\frac{1}{\Gamma^{2}(1-\alpha)}\left(\frac{T}{4}\right)^{1-2 \alpha} \frac{6 \alpha^{2}-19 \alpha+16}{(1-\alpha)^{2}(2-\alpha)(3-2 \alpha)}
$$

and

$$
\ell:=k \sqrt{\frac{C_{2}}{C_{1}}\left(A(\alpha)+\frac{2 T\|a\|_{\infty}}{3}\right)} .
$$

Moreover, for every two nonnegative constants $\gamma$ and $\sigma$ with $\gamma \neq \sigma \ell$, we set

$$
b_{\gamma}(\sigma)=\frac{\int_{0}^{T} \sup _{|\xi| \leq \gamma} F(t, \xi) \mathrm{d} t-\int_{\frac{T}{4}}^{\frac{3 T}{4}} F(t, \sigma) \mathrm{d} t}{\gamma^{2}-\ell^{2} \sigma^{2}} .
$$

We denote by $\mathscr{F}$ the class of all continuous functions $f: \mathbb{R} \rightarrow \mathbb{R}$ satisfy in the following condition:

- there exist two non-negative constants $a_{1}, a_{2}$ such that

$$
|f(t, x)| \leq a_{1}+a_{2}|x|^{p-1}, \quad \forall x \in \mathbb{R} .
$$


Theorem 3.1. Assume that $f \in \mathscr{F}$ and there exist three real constants $\gamma_{1}, \gamma_{2}$ and $\sigma$, with

$$
0<\gamma_{1}<k \sigma \ell<\gamma_{2}
$$

such that

$$
b_{\gamma_{2}}(\sigma)<b_{\gamma_{1}}(\sigma)
$$

Then, for each parameter $\lambda \in\left(\frac{C_{1}}{k^{2} b_{\gamma_{1}}(\sigma)}, \frac{C_{1}}{k^{2} b_{\gamma_{2}}(\sigma)}\right)$, problem $\left(D_{\lambda}\right)$ possesses at least one non-zero classical solution $u_{0, \lambda} \in \mathrm{E}^{\alpha}$ such that $\frac{\gamma_{1}}{C_{2}}<\left\|u_{0, \lambda}\right\|<\frac{\gamma_{2}}{C_{1}}$.

Proof. We will apply Theorem 2.8. Let $X:=\mathrm{E}^{\alpha}$ and consider the functionals $\Phi, \Psi: X \rightarrow \mathbb{R}$ defined by

$$
\Phi(u):=\frac{1}{2}\|u\|_{a, \alpha}^{2}+\sum_{j=1}^{n} J_{j}\left(u\left(t_{j}\right)\right),
$$

and

$$
\Psi(u):=\int_{0}^{T} F(t, u(t)) \mathrm{d} t
$$

From the facts $-L_{j}|\xi| \leq I_{j}(\xi) \leq L_{j}|\xi|$ for every $\xi \in \mathbb{R}, j=1, \ldots, n$, and taking (2.2) and (2.3) into account, for every $u \in X$, we have

$$
C_{1}\|u\|_{a, \alpha}^{2} \leq \Phi(u) \leq C_{2}\|u\|_{a, \alpha}^{2}
$$

Thus, the functional $\Phi: X \rightarrow \mathbb{R}$ is coercive. On the other hand, $\Phi$ and $\Psi$ are continuously Gâteaux differentiable. More precisely, we have

$$
\Phi^{\prime}(u)(v)=\int_{0}^{T}\left[\left({ }_{0}^{c} D_{t}^{\alpha} u(t)\right)\left({ }_{0}^{c} D_{t}^{\alpha} v(t)\right)+a(t) u(t) v(t)\right] \mathrm{d} t+\sum_{j=1}^{n} I_{j}\left(u\left(t_{j}\right)\right) v\left(t_{j}\right)
$$

and

$$
\Psi^{\prime}(u)(v)=\int_{0}^{T} f(t, u(t)) v(t) \mathrm{d} t
$$

for every $u, v \in X$. Fix $\lambda>0$. A critical point of the functional $J_{\lambda}:=\Phi-\lambda \Psi$ is a function $u \in X$ such that

$$
\Phi^{\prime}(u)(v)-\lambda \Psi^{\prime}(u)(v)=0, \quad \forall v \in X .
$$

Hence, the critical points of the functional $J_{\lambda}$ are weak solutions (and by Lemma 2.7 classical solutions) of the problem $\left(D_{\lambda}\right)$. At this point, let us observe that $\Phi(0)=\Psi(0)=0$. Moreover, choose $r_{1}=\frac{C_{1}}{k^{2}} \gamma_{1}^{2}$ and $r_{2}=\frac{C_{1}}{k^{2}} \gamma_{2}^{2}$. Letting $u \in \Phi^{-1}\left(-\infty, r_{1}\right)$, we find from (3.7) that

$$
\Phi^{-1}\left(-\infty, r_{1}\right)=\left\{u \in X ; \Phi(u)<r_{1}\right\} \subseteq\left\{u \in X ;|u| \leq \gamma_{1}\right\} .
$$

By same argument as above, one sees that

$$
\Phi^{-1}\left(-\infty, r_{2}\right) \subseteq\left\{u \in X ;|u| \leq \gamma_{2}\right\} .
$$

Hence, due to the condition $(\mathscr{H})$,

$$
\sup _{u \in \Phi^{-1}\left(-\infty, r_{1}\right)} \Psi(u)=\sup _{u \in \Phi^{-1}\left(-\infty, r_{1}\right)} \int_{0}^{T} F(t, u(t)) \mathrm{d} t \leq \int_{0}^{T} \sup _{|\xi| \leq \gamma_{1}} F(t, \xi) \mathrm{d} t
$$

and

$$
\sup _{u \in \Phi^{-1}\left(-\infty, r_{2}\right)} \Psi(u)=\sup _{u \in \Phi^{-1}\left(-\infty, r_{2}\right)} \int_{0}^{T} F(t, u(t)) \mathrm{d} t \leq \int_{0}^{T} \sup _{|\xi| \leq \gamma_{2}} F(t, \xi) \mathrm{d} t .
$$


Now, we define $w_{\sigma}$ by

$$
w_{\sigma}(t)= \begin{cases}\frac{4 \sigma}{T} t, & \text { if } t \in\left[0, \frac{T}{4}\right), \\ \sigma, & \text { if } t \in\left[\frac{T}{4}, \frac{3 T}{4}\right], \\ \frac{4 \sigma}{T}(T-t), & \text { if } t \in\left(\frac{3 T}{4}, T\right] .\end{cases}
$$

Clearly, $w_{\sigma} \in X$. Obviously, one has

$$
w_{\sigma}^{\prime}(t)= \begin{cases}\frac{4 \sigma}{T}, & \text { if } t \in\left(0, \frac{T}{4}\right), \\ 0, & \text { if } t \in\left(\frac{T}{4}, \frac{3 T}{4}\right), \\ -\frac{4 \sigma}{T}, & \text { if } t \in\left(\frac{3 T}{4}, T\right),\end{cases}
$$

and

$$
\begin{array}{rlr}
\left|{ }_{0}^{c} D_{t}^{\alpha} w_{\sigma}(t)\right| & =\frac{1}{\Gamma(1-\alpha)}\left(\int_{0}^{T}(t-s)^{-\alpha} w_{\sigma}^{\prime}(s) \mathrm{d} s\right) \\
& =\frac{1}{\Gamma(1-\alpha)} \begin{cases}\frac{4 \sigma}{T} \frac{t^{1-\alpha}}{1-\alpha}, & \text { if } t \in\left[0, \frac{T}{4}\right), \\
\frac{4 \sigma}{T} \frac{\left(\frac{T}{4}\right)^{1-\alpha}}{1-\alpha}, & \text { if } t \in\left[\frac{T}{4}, \frac{3 T}{4}\right], \\
\frac{4 \sigma}{T} \frac{1}{1-\alpha}\left[\left(\frac{T}{4}\right)^{1-\alpha}-\left(t-\left(\frac{3 T}{4}\right)\right)^{1-\alpha}\right], & \text { if } t \in\left(\frac{3 T}{4}, T\right],\end{cases}
\end{array}
$$

so that

$$
\left\|w_{\sigma}\right\|_{a, \alpha}^{2}=A(\alpha) \sigma^{2}+\int_{0}^{T} a(t)\left|w_{n}(t)\right|^{2} \mathrm{~d} t \leq\left(A(\alpha)+\frac{2 T\|a\|_{\infty}}{3}\right) \sigma^{2} .
$$

Using (3.7), we obtain that

$$
\Phi\left(w_{\sigma}\right) \leq\left(A(\alpha)+\frac{2 T\|a\|_{\infty}}{3}\right) C_{2} \sigma^{2}
$$

On the other hand, based on non-positivity of $J_{j}, j=1, \ldots, n$, we see that

$$
\Psi\left(w_{\sigma}\right) \geq \int_{\frac{T}{4}}^{\frac{3 T}{4}} F(t, \sigma) \mathrm{d} t
$$

Taking (3.3) into account, by a direct computation, one has $r_{1}<\Phi\left(w_{\sigma}\right)<r_{2}$. On the other hand,

$$
\begin{aligned}
\beta\left(r_{1}, r_{2}\right) & :=\sup _{v \in \Phi^{-1}\left(r_{1}, r_{2}\right)} \frac{\operatorname{su}^{-1}\left(r_{1}, r_{2}\right)}{r_{2}-\Phi(v)-\Psi(v)} \leq \frac{\sup _{u \in \Phi^{-1}\left(-\infty, r_{2}\right)} \Psi(u)-\Psi\left(w_{\sigma}\right)}{r_{2}-\Phi\left(w_{\sigma}\right)} \\
& \leq k^{2} \frac{\int_{0}^{T} \sup _{|\xi| \leq \gamma_{2}} F(t, \xi) \mathrm{d} t-\int_{\frac{T}{4}}^{\frac{3 T}{4}} F(t, \sigma) \mathrm{d} t}{C_{1} \gamma_{2}^{2}-\left(A(\alpha)+\frac{2 T\|a\|_{\infty}}{3}\right) k^{2} C_{2} \sigma^{2}},
\end{aligned}
$$

and

$$
\begin{aligned}
\rho_{2}\left(r_{1}, r_{2}\right) & :=\sup _{v \in \Phi^{-1}\left(r_{1}, r_{2}\right)} \frac{\Psi(v)-\sup _{u \in \Phi^{-1}\left(-\infty, r_{1}\right]} \Psi(u)}{\Phi(v)-r_{1}} \geq \frac{\Psi\left(w_{\sigma}\right)-\sup _{u \in \Phi^{-1}\left(-\infty, r_{1}\right]} \Psi(u)}{\Phi\left(w_{\sigma}\right)-r_{1}} \\
& \geq k^{2} \frac{\int_{0}^{T} \sup _{|\xi| \leq \gamma_{1}} F(t, \xi) \mathrm{d} t-\int_{\frac{T}{4}}^{\frac{3 T}{4}} F(t, \sigma) \mathrm{d} t}{C_{1} \gamma_{1}^{2}-\left(A(\alpha)+\frac{2 T\|a\|_{\infty}}{3}\right) k^{2} C_{2} \sigma^{2}} .
\end{aligned}
$$

Hence, by using the notation (3.1), from (3.8) and (3.9) together with (3.10), it follows that $\beta\left(r_{1}, r_{2}\right) \leq$ $k^{2} b_{\gamma_{2}}(\sigma)$ and $\rho_{2}\left(r_{1}, r_{2}\right) \geq k^{2} b_{\gamma_{1}}(\sigma)$. Finally, assumption (3.4) yields $\beta\left(r_{1}, r_{2}\right)<\rho_{2}\left(r_{1}, r_{2}\right)$. Now, from 
above the functional $\Phi$ is continuously Gâteaux differentiable while by Proposition 2.10 admits a continuous inverse on $X^{\star}$, the functional $\Phi$ is continuously Gâteaux differentiable whose Gâteaux derivative is compact and since $g \in \mathscr{G}$ the functional $\Phi-\Psi$ is coercive. Thus, from [39, Proposition 1], the functional $J_{\lambda}$ satisfies the ${ }^{\left[r_{1}\right]}(\mathrm{PS})^{\left[r_{2}\right]}$-condition for all $r_{1}$ and $r_{2}$ with $r_{1}<r_{2}<+\infty$. Therefore, by Theorem 2.8 , for each $\lambda \in\left(\frac{C_{1}}{k^{2} b_{\gamma_{1}}(\sigma)}, \frac{C_{1}}{k^{2} b_{\gamma_{2}}(\sigma)}\right), J_{\lambda}$ possesses at least one critical point $u_{0, \lambda}$ such that $r_{1}<\Phi\left(u_{0, \lambda}\right)<r_{2}$, that is, $\frac{\gamma_{1}}{C_{2}}<\left\|u_{0, \lambda}\right\|<\frac{\gamma_{2}}{C_{1}}$. This completes the proof.

Remark 3.2. The results of Theorem 3.1 hold if condition (3.2) is replaced by

$$
\text { - } \lim _{|t| \rightarrow \infty} \frac{f(t)}{|t|}=0 \text {, i.e., } f \text { is sublinear at infinity. }
$$

Now, we point out a particular case of Theorem 3.1.

Theorem 3.3. Assume that $f \in \mathscr{F}$ and there exist two positive constants $\gamma$ and $\sigma$ with $\gamma>\sigma \ell$ such that

$$
\frac{\int_{0}^{T} \sup _{|\xi| \leq \gamma} F(t, \xi) \mathrm{d} t-\int_{\frac{T}{4}}^{\frac{3 T}{4}} F(t, \sigma) \mathrm{d} t}{\gamma^{2}-\ell^{2} \sigma^{2}}<\frac{\int_{\frac{T}{4}}^{\frac{3 T}{4}} F(t, \sigma) \mathrm{d} t}{\ell^{2} \sigma^{2}}
$$

Then, for each parameter

$$
\lambda \in\left(\frac{C_{1}}{k^{2}} \frac{\ell^{2} \sigma^{2}}{\int_{\frac{T}{4}}^{\frac{3 T}{4}} F(t, \sigma) \mathrm{d} t}, \frac{C_{1}}{k^{2}} \frac{\gamma^{2}-\ell^{2} \sigma^{2}}{\int_{0}^{T} \sup _{|\xi| \leq \gamma} F(t, \xi) \mathrm{d} t-\int_{\frac{T}{4}}^{\frac{3 T}{4}} F(t, \sigma) \mathrm{d} t}\right),
$$

problem $\left(D_{\lambda}\right)$ possesses at least one non-zero classical solution $u_{0, \lambda} \in \mathrm{E}^{\alpha}$ such that $\left\|u_{0, \lambda}\right\|<\frac{\gamma}{C_{1}}$.

Proof. Taking $\gamma_{1}=0$ and $\gamma_{2}=\gamma$ and bearing (3.1) in mind, we obtain

$$
b_{\gamma}(\sigma)=\frac{\int_{0}^{T} \sup _{|\xi| \leq \gamma} F(t, \xi) \mathrm{d} t-\int_{\frac{T}{4}}^{\frac{3 T}{4}} F(t, \sigma) \mathrm{d} t}{\gamma^{2}-\ell^{2} \sigma^{2}}<\frac{\int_{\frac{T}{4}}^{\frac{3 T}{4}} F(t, \sigma) \mathrm{d} t}{\ell^{2} \sigma^{2}}=b_{0}(\sigma) .
$$

Hence, Theorem 3.1 ensures the conclusion.

Now, we give an application of Theorem 2.9 which will be used later to obtain multiple solutions for the problem $\left(D_{\lambda}\right)$.

Theorem 3.4. Assume that $f \in \mathscr{F}$ and there exist two positive constants $\bar{\gamma}$ and $\bar{\sigma}$ with $\bar{\gamma}<\bar{\sigma} \ell$ such that $\alpha_{0} G(\bar{\sigma})<|\alpha|_{1} G(\bar{\gamma})$ Then, for each $\lambda>\tilde{\lambda}$, where

$$
\tilde{\lambda}:=\frac{C_{1}}{k^{2}} \frac{\bar{\gamma}^{2}-\ell^{2} \bar{\sigma}^{2}}{\int_{0}^{T} \sup _{|\xi| \leq \bar{\gamma}} F(t, \xi) \mathrm{d} t-\int_{\frac{T}{4}}^{\frac{3 T}{4}} F(t, \bar{\sigma}) \mathrm{d} t},
$$

problem $\left(D_{\lambda}\right)$ possesses at least one non-trivial classical solution $\bar{u}_{0, \lambda} \in \mathrm{E}^{\alpha}$ such that $\left\|\bar{u}_{0, \lambda}\right\|>\frac{\bar{\gamma}}{C_{2}}$.

Proof. Take $X=\mathrm{E}^{\alpha}$ and put $I_{\lambda}=\Phi-\lambda \Psi$, where $\Phi$ and $\Psi$ are given as in (3.5) and (3.6), respectively. The functionals $\Phi$ and $\Psi$ satisfy all assumptions requested in Theorem 2.9. Put $\bar{r}:=\frac{C_{1}}{k^{2}} \bar{\gamma}^{2}$. From [39, Proposition 1], the functional $J_{\lambda}$ satisfies ${ }^{[\bar{r}]}$ (PS) ${ }^{[r]}$-condition for all $r$ with $r>\bar{r}$. Arguing as in the proof 
of Theorem 3.1, we obtain that

$$
\begin{aligned}
\rho(\bar{r}) & =\sup _{v \in \Phi^{-1}(\bar{r},+\infty)} \frac{\Psi(v)-\sup _{u \in \Phi^{-1}(-\infty, \bar{r}]} \Psi(u)}{\Phi(v)-\bar{r}} \\
& \geq \frac{\Psi\left(w_{\sigma}\right)-\sup _{u \in \Phi^{-1}(-\infty, \bar{r}]} \Psi(u)}{\Phi\left(w_{\sigma}\right)-\bar{r}} \\
& \geq \frac{k^{2}}{C_{1}} \frac{\int_{0}^{T} \sup _{|\xi| \leq \bar{\gamma}} F(t, \xi) \mathrm{d} t-\int_{\frac{T}{4}}^{\frac{3 T}{4}} F(t, \bar{\sigma}) \mathrm{d} t}{\bar{\gamma}^{2}-\ell^{2} \bar{\sigma}^{2}} .
\end{aligned}
$$

Hence, from our assumption, it follows that $\rho(\bar{r})>0$. Therefore, it follows from Theorem 2.9 with $\beta^{*}=$ 0 , for each $\lambda>\tilde{\lambda}$, the functional $J_{\lambda}$ admits at least one local minimum $\bar{u}_{0, \lambda} \in \mathrm{E}^{\alpha}$ such that $\Phi\left(\bar{u}_{0, \lambda}\right)>\bar{r}$, which is just $\left\|\bar{u}_{0, \lambda}\right\|>\frac{\bar{\gamma}}{C_{2}}$. Thus the conclusion is obtained.

The following result is a straight consequence of Theorem 3.3.

Theorem 3.5. Assume that $f \in \mathscr{F}$ and

$$
\lim _{\xi \rightarrow 0^{+}} \frac{F(t, \xi)}{\xi^{2}}=+\infty
$$

Furthermore, let $\gamma>0$ and set $\lambda_{\gamma}^{\star}:=\frac{C_{1}}{k^{2}} \frac{\gamma^{2}}{\int_{0}^{T} \sup _{|\xi| \leq \gamma} F(t, \xi) \mathrm{d} t}$. Then, for every $\lambda \in\left(0, \lambda_{\gamma}^{\star}\right)$, problem $\left(D_{\lambda}\right)$ admits at least one non-zero classical solution $u_{0, \lambda} \in \mathrm{E}^{\alpha}$ such that $\left\|u_{0, \lambda}\right\|<\frac{\gamma}{C_{1}}$.

Proof. Fix $\lambda \in\left(0, \lambda_{\gamma}^{\star}\right)$. From (3.11), there exists a constant $\sigma>0$ with $\gamma>\sigma \ell$ such that

$$
\frac{C_{1}}{k^{2}} \frac{\ell^{2} \sigma^{2}}{\int_{\frac{T}{4}}^{\frac{3 T}{4}} F(t, \sigma) \mathrm{d} t}<\lambda<\frac{C_{1}}{k^{2}} \frac{\gamma^{2}-\ell^{2} \sigma^{2}}{\int_{0}^{T} \sup _{|\xi| \leq \gamma} F(t, \xi) \mathrm{d} t-\int_{\frac{T}{4}}^{\frac{3 T}{4}} F(t, \sigma) \mathrm{d} t} .
$$

Hence, by Theorem 3.3, problem $\left(D_{\lambda}\right)$ possesses at least one non-zero classical solution $u_{0, \lambda}$ such that $\left\|u_{0, \lambda}\right\|<\frac{\gamma}{C_{1}}$.

Example 3.6. Consider the problem

$$
\begin{gathered}
{ }_{t} D_{1}^{\frac{2}{3}}\left({ }_{0}^{c} D_{t}^{\frac{2}{3}} u(t)\right)+u(t)=\lambda t f(u(t)), \quad t \neq \frac{1}{3}, \frac{2}{3}, \text { a.e. } t \in[0,1], \\
\Delta\left({ }_{t} D_{1}^{-\frac{1}{3}}\left({ }_{0}^{c} D_{t}^{\frac{2}{3}} u\right)\right)\left(\frac{1}{3}\right)=\frac{\lambda}{9} \Gamma^{2}\left(\frac{2}{3}\right) \sin \left(u\left(\frac{1}{3}\right)\right), \\
\Delta\left({ }_{t} D_{1}^{-\frac{1}{3}}\left({ }_{0}^{c} D_{t}^{\frac{2}{3}} u\right)\right)\left(\frac{2}{3}\right)=\frac{\lambda}{12} \Gamma^{2}\left(\frac{2}{3}\right) \arctan \left(u\left(\frac{1}{3}\right)\right), \\
u(0)=u(1)=0
\end{gathered}
$$

with

$$
f(x)= \begin{cases}e^{x}, & x \in(-\infty,-1], \\ e^{\sin \left(\frac{\pi}{2} x\right)}, & x \in(-1,1), \\ e^{-\cos (\pi x)}, & x \in[1, \infty) .\end{cases}
$$

Direct calculations show that $k=\frac{3}{\sqrt{3} \Gamma\left(\frac{2}{3}\right)}$ and $C_{1}=\frac{29}{72}$ and

$$
\lim _{\xi \rightarrow 0^{+}} \frac{f(\xi)}{\xi}=\lim _{\xi \rightarrow 0^{+}} \frac{e^{\sin \left(\frac{\pi}{2} \xi\right)}}{\xi}=+\infty, \quad \lim _{\xi \rightarrow+\infty} \frac{f(\xi)}{\xi}=\lim _{\xi \rightarrow+\infty} \frac{e^{-\cos (\pi \xi)}}{\xi}=0 .
$$


Choosing $\gamma=1$, we clearly see that all assumptions of Theorem 3.5 are satisfied. Hence, applying Theorem 3.5 and Remark 3.2 for every $\lambda \in\left(0, \frac{58}{216 e} \Gamma^{2}\left(\frac{2}{3}\right)\right)$, we see that problem (3.12) possesses at least one non-zero classical solution $u_{0, \lambda} \in \mathrm{E}^{\alpha}$ such that $\left\|u_{0, \lambda}\right\|<\frac{72}{29}$.

Theorem 3.7. Suppose that $g \in \mathscr{G}$. Then the mapping $\lambda \mapsto J_{\lambda}\left(u_{0, \lambda}\right)$ is negative and strictly decreasing in $\left(0, \lambda_{\gamma}^{\star}\right)$.

Proof. The restriction of the functional $J_{\lambda}$ to $\Phi^{-1}(0, r)$ admits a global minimum, which is a critical point (local minimum) of $J_{\lambda}$ in $\mathrm{E}^{\alpha}$. Moreover, since $w_{\sigma} \in \Phi^{-1}(0, r)$ and

$$
\frac{\Phi\left(w_{\sigma}\right)}{\Psi\left(w_{\sigma}\right)} \leq \frac{\left(A(\alpha)+\frac{2 T\|a\|_{\infty}}{3}\right) C_{2} \sigma^{2}}{\int_{\frac{T}{4}}^{\frac{3 T}{4}} F(t, \sigma) \mathrm{d} t .}<\lambda
$$

we have

$$
J_{\lambda}\left(u_{0, \lambda}\right) \leq J_{\lambda}\left(w_{\sigma}\right)=\Phi\left(w_{\sigma}\right)-\lambda \Psi\left(w_{\sigma}\right)<0 .
$$

Next, we observe that $J_{\lambda}(u)=\lambda\left(\frac{\Phi(u)}{\lambda}-\Psi(u)\right)$ for every $u \in \mathrm{E}^{\alpha}$ and fix $0<\lambda_{1}<\lambda_{2}<\lambda_{\gamma}^{\star}$. Set

$$
m_{\lambda_{1}}:=\left(\frac{\Phi\left(u_{0, \lambda_{1}}\right)}{\lambda_{1}}-\Psi\left(u_{0, \lambda_{1}}\right)\right)=\inf _{u \in \Phi^{-1}\left(0, r_{2}\right)}\left(\frac{\Phi(u)}{\lambda_{1}}-\Psi(u)\right),
$$

and

$$
m_{\lambda_{2}}:=\left(\frac{\Phi\left(u_{0, \lambda_{2}}\right)}{\lambda_{2}}-\Psi\left(u_{0 \lambda_{2}}\right)\right)=\inf _{u \in \Phi^{-1}\left(0, r_{2}\right)}\left(\frac{\Phi(u)}{\lambda_{2}}-\Psi(u)\right) .
$$

Clearly, as claimed before, $m_{\lambda_{i}}<0$ (for $i=1,2$ ), and $m_{\lambda_{2}} \leq m_{\lambda_{1}}$ thanks to $\lambda_{1}<\lambda_{2}$. Hence, $\lambda \mapsto J_{\lambda}\left(u_{0, \lambda}\right)$ is strictly decreasing in $\left(0, \lambda_{\gamma}^{\star}\right)$ since the fact

$$
J_{\lambda_{2}}\left(u_{0, \lambda_{2}}\right)=\lambda_{2} m_{\lambda_{2}} \leq \lambda_{2} m_{\lambda_{1}}<\lambda_{1} m_{\lambda_{1}}=J_{\lambda_{1}}\left(u_{0, \lambda_{1}}\right)
$$

Remark 3.8. Generally, Theorem 3.5 ensures that if $g \in \mathscr{G}$ satisfies (3.11), then for every parameter $\lambda$ belonging to the real interval $\Lambda_{\Omega}:=\left(0, \lambda^{\star}\right)$, where $\lambda^{\star}:=\frac{C_{1}}{k^{2}} \sup _{\gamma>0} \frac{\gamma^{2}}{F(t, \gamma)},\left(D_{\lambda}\right)$ possesses at least one non-zero solution $u_{0, \lambda} \in \mathrm{E}^{\alpha}$.

Remark 3.9. We note that, in particular, if $f$ is sublinear at infinity with respect to the second variable, then Theorem 3.5 ensures that problem $\left(D_{\lambda}\right)$ admits at least one non-zero classical solution for every positive parameter $\lambda$. Moreover, in our case, the obtained solution is non-zero, while the classical direct method approach, that can be accept in this context, ensures the existence of at least one solution that may be zero.

Remark 3.10. A careful analysis of the proof of Theorem 3.5 ensures that the result still remains true if condition (3.11) is replaced by the more general assumption $\limsup _{\xi \rightarrow 0^{+}} \frac{F(t, \xi)}{\xi^{2}}=+\infty$. Moreover, the previous asymptotic condition at zero can be replaced by the following form

$$
\limsup _{\xi \rightarrow 0^{+}} \frac{f(t, \xi)}{\xi}=+\infty \text {. }
$$

Therefore, it is natural to obtain the following result. 
Theorem 3.11. Let $\lim _{\xi \rightarrow 0^{+}} \frac{f(t, \xi)}{\xi}=+\infty$ and $\lim _{\xi \rightarrow+\infty} \frac{f(t, \xi)}{\xi}=0$. Then there exists $\lambda^{\star}>0$ such that, for every $\lambda \in\left(0, \lambda^{\star}\right),\left(D_{\lambda}\right)$ possesses at least one non-zero classical solution $u_{0, \lambda} \in \mathrm{E}^{\alpha}$. Moreover, we have

$$
\left(\int_{0}^{T}\left(\left|{ }_{0}^{c} D_{t}^{\alpha} u(t)\right|^{2} d t+a(t)|u(t)|^{2}\right) \mathrm{d} t\right)^{\frac{1}{2}} \rightarrow 0
$$

as $\lambda \rightarrow 0^{+}$and the mapping

$$
\lambda \mapsto\left(\int_{0}^{T}\left(\left.{ }_{0}^{c} D_{t}^{\alpha} u(t)\right|^{2} \mathrm{~d} t+a(t)|u(t)|^{2}\right) \mathrm{d} t\right)^{\frac{1}{2}}-\int_{0}^{T}\left(\int_{0}^{u_{0, \lambda}} f(t, x) \mathrm{d} t\right) \mathrm{d} x
$$

is negative and strictly decreasing in $\left(0, \lambda^{\star}\right)$.

Below, we show how the former analysis can be used to pass from the existence of at least one nontrivial solution to that of at least two nontrivial solutions. Accordingly, we start with the following theorem, where the celebrated Ambrosetti-Rabinowitz condition is necessary.

Theorem 3.12. Let $g$ be a continuous function such that $g(0) \neq 0$ and assumption (3.13) holds. Furthermore, assume that

(AR) there are constants $v>2$ and $\rho>0$ such that, for all $\xi \geq \rho$, one has

$$
0<v F(t, \xi) \leq \xi f(t, \xi) \text {. }
$$

Then, for each $\lambda \in \Lambda_{\Omega}$, problem $\left(D_{\lambda}\right)$ admits at least two non-trivial classical solutions in the space $\mathrm{E}^{\alpha}$.

Proof. Fix $\lambda \in \Lambda_{\Omega}$. In view of the assumption (3.13), Theorem 3.5 ensures that problem $\left(D_{\lambda}\right)$ admits at least one weak non-zero solution $u_{1}$ in $\mathrm{E}^{\alpha}$, which is a local minimum of the functional $J_{\lambda}$ as defined in the proof of Theorem 3.1. Now, we prove the existence of the second local minimum distinct from the first one. To this end, we verify the hypotheses of the mountain-pass theorem for $J_{\lambda}$. Clearly, $J_{\lambda}$ is of class $\mathrm{C}^{1}$ and $J_{\lambda}(0)=0$. The first part of proof guarantees that $u_{1} \in \mathrm{E}^{\alpha}$ is a nontrivial local minimum for $J_{\lambda}$ in $\mathrm{E}^{\alpha}$. We can assume that $u_{1}$ is a strict local minimum for $J_{\lambda}$ in $\mathrm{E}^{\alpha}$. Therefore, there is $\rho>0$ such that $\inf _{\left\|u-u_{1}\right\|=\rho} I_{\lambda}(u)>I_{\lambda}\left(u_{1}\right)$. So condition [38, $\left(I_{1}\right)$, Theorem 2.2] is verified. By integrating the condition (3.14), there exist constants $a_{1}, a_{2}>0$ such that $F(t, x) \geq a_{1}|x|^{v}-a_{2}$ for all $x \in \mathbb{R}$. Now, choosing any $u \in \mathrm{E}^{\alpha}$, one find that

$$
\begin{aligned}
J_{\lambda}(\tau u) & =(\Phi-\lambda \Psi)(\tau u) \leq C_{2}\|\tau u\|_{a, \alpha}^{2}-\lambda \int_{\mathbb{R}} F(t, \tau u(x)) \mathrm{d} x \\
& \leq C_{2} \tau^{2}\|u\|_{a, \alpha}^{2}-\lambda \tau^{v} a_{1} \int_{0}^{T}|u(x)|^{v} \mathrm{~d} x+\lambda a_{2}|\alpha|_{1} \rightarrow-\infty, \quad \tau \rightarrow+\infty
\end{aligned}
$$

Thus condition [38, $\left(I_{2}\right)$, Theorem 2.2] is satisfied. Therefore, $J_{\lambda}$ satisfies the geometry of mountain pass. Moreover, $J_{\lambda}$ satisfies the (PS)-condition. Indeed, assume that $\left\{u_{n}\right\}_{n \in \mathbb{N}} \subset X$ such that $\left\{J_{\lambda}\left(u_{n}\right)\right\}_{n \in \mathbb{N}}$ is bounded and $J_{\lambda}^{\prime}\left(u_{n}\right) \rightarrow 0$ as $n \rightarrow+\infty$. Then, there exists a positive constant $c_{0}$ such that

$$
\left|J_{\lambda}\left(u_{n}\right)\right| \leq c_{0},\left|J_{\lambda}^{\prime}\left(u_{n}\right)\right| \leq c_{0} \text { for all } n \in \mathbb{N} .
$$

Therefore, we infer to deduce from the definition of $J_{\lambda}^{\prime}$ and the assumption $(A R)$ that

$$
\begin{aligned}
& c_{0}+c_{1}\left\|u_{n}\right\| \geq v J_{\lambda}\left(u_{n}\right)-J_{\lambda}^{\prime}\left(u_{n}\right)\left(u_{n}\right) \geq\left(\frac{v}{2}-1\right)\left\|u_{n}\right\|_{a, \alpha}^{2} \\
& -\lambda \int_{\Omega}\left(v F\left(t, u_{n}(t)\right)-f\left(t, u_{n}(t)\right)\left(u_{n}(t)\right)\right) \mathrm{d} t \geq\left(\frac{v}{2}-1\right)\left\|u_{n}\right\|_{a, \alpha}^{2},
\end{aligned}
$$


for some $c_{1}>0$. Since $v>2$, we find that $\left(u_{n}\right)$ is bounded. This implies that $\left\{u_{n}\right\}$ converges strongly to $u$ in $\mathrm{E}^{\alpha}$. Consequently, $J_{\lambda}$ satisfies (PS)-condition. Hence, by the classical theorem of Ambrosetti and Rabinowitz [41, Theorem 5.8], we establish a critical point $u_{2}$ of $J_{\lambda}$ such that $J_{\lambda}\left(u_{2}\right)>J_{\lambda}\left(u_{1}\right)$. Since $g(0) \neq 0, u_{1}$ and $u_{2}$ are two distinct non-trivial classical solutions of $\left(D_{\lambda}\right)$. The proof is completed.

Remark 3.13. The non-triviality of the second solution ensured by Theorem 3.12 can be achieved also in the case $g(0)=0$ requiring the extra conditions at zero

$$
\limsup _{\xi \rightarrow 0^{+}} \frac{F(t, \xi)}{|\xi|^{2}}=+\infty \quad \text { and } \quad \liminf _{\xi \rightarrow 0^{+}} \frac{F(t, \xi)}{|\xi|^{2}}>-\infty .
$$

Indeed, let $0<\bar{\lambda}<\lambda^{*}$, where $\lambda^{*}=\frac{C_{1}}{k^{2}} \sup _{\gamma>0} \frac{\gamma^{2}}{F(t, \gamma)}$. Then there exists $\bar{\gamma}>0$ such that $\frac{C_{1} \bar{\lambda}}{k^{2}}<\frac{\bar{\gamma}^{p}}{F(t, \gamma)}$. Let $\Phi$ and $\Psi$ be as given in (3.5) and (3.6), respectively. Due to Theorem 3.12, for every $\lambda \in(0, \bar{\lambda})$, there exists a critical point of $J_{\lambda}=\Phi-\lambda \Psi$ such that $u_{\lambda} \in \Phi^{-1}\left(-\infty, r_{\lambda}\right)$, where $r_{\lambda}:=\frac{\bar{\gamma}^{2}}{p}$. In particular, $u_{\lambda}$ is a global minimum of the restriction of $J_{\lambda}$ to $\Phi^{-1}\left(-\infty, r_{\lambda}\right)$. We will prove that $u_{\lambda}$ cannot be trivial. Let us show that

$$
\limsup _{\|u\| \rightarrow 0^{+}} \frac{\Psi(u)}{\Phi(u)}=+\infty .
$$

In view of (3.15), we can consider a sequence $\left\{\xi_{n}\right\} \subset \mathbb{R}^{+}$converging to zero and two constants $\imath, \kappa$ (with $\imath>0$ ) such that $\lim _{n \rightarrow+\infty} \frac{F\left(t, \xi_{n}\right)}{\left|\xi_{n}\right|^{p}}=+\infty$ and $F(t, \xi) \geq \kappa|\xi|^{\alpha p}$, for every $\xi \in[0, l]$. We consider a set $\mathscr{F} \subset B$ of positive measure and a function $v \in \mathrm{E}^{\alpha}$ such that

$(k 1) v(t) \in[0,1]$ for every $t \in[0, T]$;

(k2) $v(t)=1$ for every $t \in \mathscr{F}$.

Hence, fix $N>0$ and consider a real positive number $\eta$ with

$$
N<\frac{2 \eta \operatorname{meas}(\mathscr{F})+2 \kappa \int_{\mathbb{R} \backslash \mathscr{F}}|v(t)|^{2} \mathrm{~d} t}{\|v\|_{a, \alpha}^{2}} .
$$

Then there is $n_{0} \in \mathbb{N}$ such that $\xi_{n}<\imath$ and $F\left(t, \xi_{n}\right) \geq \eta\left|\xi_{n}\right|^{2}$, for every $n>n_{0}$. Now, for every $n>n_{0}$, by the properties of the function $v$ (that is, $0 \leq \xi_{n} v(t)<\imath$ for $n$ large enough), one has

$$
\begin{aligned}
\frac{\Psi\left(\xi_{n} v\right)}{\Phi\left(\xi_{n} v\right)} & =\frac{\int_{\mathscr{F}} F\left(t, \xi_{n}\right) \mathrm{d} t+\int_{[0, T] \backslash \mathscr{F}} F\left(t, \xi_{n} v(t)\right) \mathrm{d} t}{\Phi\left(\xi_{n} v\right)} \\
& >\frac{2 \eta \operatorname{meas}(\mathscr{F})+2 \kappa \int_{\mathbb{R} \backslash \mathscr{F}}|v(t)|^{2} \mathrm{~d} t}{\|v\|_{a, \alpha}^{2}}>N .
\end{aligned}
$$

Since $N$ could be arbitrarily large, we get $\lim _{n \rightarrow \infty} \frac{\Psi\left(\xi_{n} v\right)}{\Phi\left(\xi_{n} v\right)}=+\infty$, from which (3.16) clearly follows. So, there exists a sequence $\left\{\omega_{n}\right\} \subset X$ strongly converging to zero such that, for $n$ large enough, $\omega_{n} \in$ $\Phi^{-1}\left(-\infty, r_{\lambda}\right)$ and

$$
J_{\lambda}\left(\omega_{n}\right)=\Phi\left(\omega_{n}\right)-\lambda \Psi\left(\omega_{n}\right)<0 .
$$

Since $u_{\lambda}$ is a global minimum of the restriction of $J_{\lambda}$ to $\Phi^{-1}\left(-\infty, r_{\lambda}\right)$, we obtain $J_{\lambda}\left(u_{\lambda}\right)<0$, so that $u_{\lambda}$ is not trivial.

Below, we present one application of Theorem 3.12 as follows. 
Example 3.14. Let $n=1, \alpha=\frac{3}{4}, T=\pi, a(t)=e^{t}$ for all $t \in[0, \pi], I_{1}(x)=\frac{\Gamma^{2}\left(\frac{3}{4}\right)}{2} \ln \left(1+x^{2}\right)$ for all $x \in \mathbb{R}, f(t, x)=t\left(1+x^{6}\right)$ for all $(t, x) \in[0, \pi] \times \mathbb{R}$. Thus $L_{1}=\frac{\Gamma^{2}\left(\frac{3}{4}\right)}{4}, k=\frac{2}{\sqrt{2} \Gamma\left(\frac{3}{4}\right)}$ and $C_{1}=\frac{1}{4}$. Moreover, $f(0)=1 \neq 0, \lim _{\xi \rightarrow 0^{+}} \frac{f(\xi)}{\xi^{p-1}}=\lim _{\xi \rightarrow 0^{+}} \frac{1+\xi^{6}}{\xi^{2}}=+\infty$ and taking into account that

$$
\lim _{|\xi| \rightarrow+\infty} \frac{\xi f(\xi)}{F(\xi)}=\lim _{|\xi| \rightarrow+\infty} \frac{\xi+\xi^{7}}{\xi+\frac{1}{7} \xi^{7}}=7>3=p,
$$

by choosing $v=7>3=p$, there exist $\rho>1$ such that the assumption (AR) in Theorem 3.12 is fulfilled for all $|\xi|>\rho$. Hence, by applying Theorem 3.12 and Remark 3.2, for every $\lambda>0,\left(D_{\lambda}\right)$ in this case possesses at least two nontrivial classical solutions.

Finally, as a consequence of Theorems 3.3 and 3.4, we can obtain the following existence result of three solutions.

Theorem 3.15. Assume that $g(0) \neq 0$ and there exist four positive constants $\gamma, \sigma, \bar{\gamma}$ and $\bar{\sigma}$ with $\bar{\gamma}<\bar{\sigma} \ell \leq$ $\sigma<\gamma$ such that $\int_{0}^{T} \sup _{|\xi| \leq \gamma} F(t, \xi) \mathrm{d} t<\int_{\frac{T}{4}}^{\frac{3 T}{4}} F(t, \sigma) \mathrm{d} t$ and $\int_{0}^{T} \sup _{|\xi| \leq \bar{\gamma}} F(t, \xi) \mathrm{d} t<\int_{\frac{T}{4}}^{\frac{3 T}{4}} F(t, \bar{\sigma}) \mathrm{d} t$ hold, and

$$
\frac{\int_{0}^{T} \sup _{|\xi| \leq \gamma} F(t, \xi) \mathrm{d} t}{\gamma^{2}}<\frac{\int_{0}^{T} \sup _{|\xi| \leq \gamma} F(t, \xi) \mathrm{d} t-\int_{\frac{T}{4}}^{\frac{3 T}{4}} F(t, \sigma) \mathrm{d} t}{\gamma^{2}-\ell^{2} \sigma^{2}}
$$

is satisfied. Then, for each

$$
\lambda \in \Lambda=\left(\max \left\{\tilde{\lambda}, \frac{\gamma^{2}-\ell^{2} \sigma^{2}}{\int_{0}^{T} \sup _{|\xi| \leq \gamma} F(t, \xi) \mathrm{d} t-\int_{\frac{T}{4}}^{\frac{3 T}{4}} F(t, \sigma) \mathrm{d} t}\right\}, \frac{\gamma^{2}}{\int_{0}^{T} \sup _{|\xi| \leq \gamma} F(t, \xi) \mathrm{d} t}\right),
$$

problem $\left(D_{\lambda}\right)$ possesses at least three classical solutions $u_{0, \lambda}, \bar{u}_{0, \lambda}$ and $\tilde{u}_{0, \lambda}$ such that $\left\|u_{0, \lambda}\right\|<\frac{\gamma}{C_{1}}$ and $\left\|\bar{u}_{0, \lambda}\right\|>\frac{\bar{\gamma}}{C_{2}}$

Proof. First, in view of (3.17), we have $\Lambda \neq \emptyset$. Next, we fix $\lambda \in \Lambda$. Employing Theorem 3.3, there is a positive classical solution $u_{0, \lambda}$ such that $\left\|u_{0, \lambda}\right\|<\frac{\gamma}{C_{1}}$, which is a local minimum for the associated functional $J_{\lambda}$, while Theorem 3.4 ensures a weak solution $\bar{u}_{0, \lambda}$ such that $\left\|\bar{u}_{0, \lambda}\right\|>\frac{\bar{\gamma}}{C_{2}}$, which is a local minimum for $J_{\lambda}$. Arguing as in the proof of Theorem 3.1, we observe that $J_{\lambda}$ is coercive. Then it satisfies the (PS)-condition. Hence, the conclusion follows from the mountain pass theorem as given by Pucci and Serrin [42].

\section{REFERENCES}

[1] H. Risken, The Fokker-Planck Equation, Springer, Berlin, 1988.

[2] D. Benson, S. Wheatcraft, M. Meerschaert, Application of a fractional advection dispersion equation, Water Resour. Res. 36 (2000), 1403-1412.

[3] D. Benson, S. Wheatcraft, M. Meerschaert, The fractional-order governing equation of Lévy motion, Water Resour. Res. 36 (2000), 1413-1423.

[4] J. Eggleston, S. Rojstaczer, Identification of large-scale hydraulic conductivity trends and the influence of trends on contaminant transport, Water Resour. Res. 34 (1998), 2155-2168.

[5] S.G. Samko, A.A. Kilbas, O.I. Marichev, Fractional Integral and Derivatives: Theory and Applications, Gordon and Breach, Longhorne, PA, 1993.

[6] R. Hilferm, Applications of Fractional Calculus in Physics, World Scientific, Singapore, 2000.

[7] A.A. Kilbas, H.M. Srivastava, J.J. Trujillo, Theory and Applications of Fractional Differential Equations, Elsevier, Amsterdam, 2006. 
[8] F. Metzler, W. Schick, H. G. Kilan, T. F. Nonnenmacher, Relaxation in filled polymers: A Fractional Calculus approach, J. Chem. Phys. 103 (1995) 7180-7186.

[9] Y. Zhou, Basic theory of fractional differential equations, Vol. 6. Singapore, World Scientific, 2014.

[10] K.S. Miller, B. Ross, An Introduction to the Fractional Calculus and Fractional Differential Equations, Wiley, New York, 1993.

[11] I. Podlubny, Fractional Differential Equations, Academic Press, San Diego, 1999.

[12] M. Ferrara, G.M. Bisci, Remarks for one-dimensional fractional equations, Opuscula Math.34 (2014), 691-698.

[13] M. Galewski, G.M. Bisci, Existence results for one-dimensional fractional equations, Math. Meth. Appl. Sci. 39 (2016), 1480-1492.

[14] J.R. Graef, L. Kong, Q. Kong, Multiple solutions of systems of fractional boundary value problems, Appl. Anal. 94 (2015), 1288-1304.

[15] J.R. Graef, L. Kong, Q. Kong, M. Wang, Fractional boundary value problems with integral boundary conditions, Appl. Anal. 92 (2013), 2008-2020.

[16] L. Kong, Existence of solutions to boundary value problems arising from the fractional advection dispersion equation, Electron. J. Diff. Equ. 2013 (2013), Article ID 106.

[17] V. Lakshmikantham, D.D. Bainov, P.S. Simeonov, Theory of impulsive differential equations, Series in Modern Applied Mathematics, vol.6, World Scientific, Teaneck, NJ, 1989.

[18] D. Bainov, P. Simeonov, Systems with Impulse Effect, Ellis Horwood Series: Mathematics and Its Applications, Ellis Horwood, Chichester, 1989.

[19] S. Heidarkhani, M. Ferrara, A. Salari, Infinitely many periodic solutions for a class of perturbed second-order differential equations with impulses, Acta Appl. Math. 139 (2015), 81-94.

[20] A.M. Samoilenko, N.A. Perestyuk, Impulsive differential equations, World Scientific, Singapore, 1995.

[21] G. Bonanno, R. Rodríguez-López, S. Tersian, Existence of solutions to boundary-value problem for impulsive fractional differential equations, Fract. Calc. Appl. Anal. 3 (2014), 717-744.

[22] M. De la Sen, On Riemann-Liouville and Caputo impulsive fractional calculus, In Proc. of the World Congress on Engineering 2011, volume 1, London, UK, 2011.

[23] Y. Tian, Z. Bai, Existence results for the three-point impulsive boundary value problem involving fractional differential equations, Comput. Math. Appl. 59 (2010), 2601-2609.

[24] E. Ait Dads, M. Benchohra, S. Hamani, Impulsive fractional differential inclusions involving fractional derivative, Fract. Calc. Appl. Anal. 12 (2009), 15-38.

[25] C. Bai, Impulsive periodic boundary value problems for fractional differential equation involving Riemann-Liouville sequential fractional derivative, J. Math. Anal. Appl. 384 (2011), 211-231.

[26] C. Bai, Solvability of multi-point boundary value problem of nonlinear impulsive fractional differential equation at resonance, Electron. J. Qual. Theory Differ. Equ. 89 (2011), 1-19.

[27] T.D. Ke, D. Lan, Decay integral solutions for a class of impulsive fractional differential equations in Banach spaces, Fract. Calc. Appl. Anal. 17 (2014), 96-121.

[28] R. Rodríguez-López, S. Tersian, Multiple solutions to boundary value problem for impulsive fractional differential equations, Fract. Calc. Appl. Anal. 17 (2014), 1016-1038.

[29] S. Heidarkhani, A. Salari, Nontrivial solutions for impulsive fractional differential systems through variational methods, Comput. Math. Appl. (2016), http://dx.doi.org/10.1016/j.camwa.2016.04.016.

[30] S. Heidarkhani, Y. Zhao, G. Caristi, G.A. Afrouzi, S. Moradi, Infinitely many solutions for perturbed impulsive fractional differential systems, Appl. Anal. 96 (2017), 1401-1424.

[31] S. Heidarkhani, Y. Zhou, G. Caristi, G.A. Afrouzi, S. Moradi, Existence results for fractional differential systems through a local minimization principle, Comput. Math. Appl. (2016), http://dx.doi.org/10.1016/j.camwa.2016.04.012.

[32] Y. Zhao, H. Chen, B. Qin, Multiple solutions for a coupled system of nonlinear fractional differential equations via variational methods, Appl. Math. Comput. 257 (2015), 417-427.

[33] Y. Zhao, H. Chen, Q. Zhang, Infinitely many solutions for fractional differential system via variational method, J. Appl. Math. Comput. 50 (2016), 589-609. 
[34] J. Chu, S. Heidarkhani, A. Salari, G. Caristi, Weak solutions and energy estimates for singular $p$-Laplacian type equations, J. Dyn. Control Syst. 24 (2018), 51-63.

[35] G. D’Aguì, Existence results for a mixed boundary value problem with Sturm-Liouville equation, Adv. Pure Appl. Math. 2 (2011), 237-248.

[36] F. Jiao, Y. Zhou, Existence of solutions for a class of fractional boundary value problems via critical point theory, Comput. Math. Appl. 62 (2011), 1181-1199.

[37] D. Motreanu, V. Rădulescu, Variational and Non-variational Methods in Nonlinear Analysis and Boundary Value Problems, Nonconvex Optimization and Applications, Kluwer Academic Publishers, 2003.

[38] P.H. Rabinowitz, Minimax Methods in Critical Point Theory with Applications to Differential Equations, CBMS Reg. Conf. Ser. Math., vol. 65, American Mathematical Society, Providence, RI, 1986.

[39] G. Bonanno, A critical point theorem via the Ekeland variational principle, Nonlinear Anal. 75 (2012), 2992-3007.

[40] E. Zeidler, Nonlinear Functional Analysis and its Applications, Vol. III, Springer, New York, 1985.

[41] A. Ambrosetti, P.H. Rabinowitz, Dual variational methods in critical point theory and applications, J. Funct. Anal. 14 (1973), 349-381.

[42] P. Pucci, J. Serrin, A mountain pass theorem, J. Differential Equations 60 (1985), 142-149. 